COMUNICAÇÃO CIENTÍFICA

\title{
CARACTERÍSTICAS AGRONÔMICAS DE SEIS GENÓTIPOS DE MARACUJAZEIRO-AZEDO CULTIVADOS NO DISTRITO FEDERAL ${ }^{1}$
}

\author{
SIMONE DE PAULA MIRANDA ABREU², JOSÉ RICARDO PEIXOTO, \\ NILTON TADEU VILELA JUNQUEIRA ${ }^{4}$, MARCELO ALVES DE FIGUEIREDO SOUSA ${ }^{5}$
}

RESUMO - Este trabalho teve como objetivo avaliar as características agronômicas de seis genótipos de maracujazeiro-azedo cultivados no Distrito Federal. O experimento foi desenvolvido na área experimental da Fazenda Água Limpa da Universidade de Brasília, no DF. Utilizou-se o delineamento em blocos casualizados, com quatro repetições, seis tratamentos e sete plantas por parcela. Foram avaliados os genótipos: Rubi Gigante, EC-3-0, EC-L-7, RC-3, Redondão e Gigante Amarelo. Os parâmetros analisados, durante seis meses (17 colheitas), foram: produtividade total estimada, quantidade de frutos e coloração da casca. O genótipo Rubi Gigante teve a maior produtividade $\left(16,69 \mathrm{t} / \mathrm{ha}^{-1}\right)$, enquanto o RC-3 a menor $\left(2,92 \mathrm{t} / \mathrm{ha}^{-1}\right)$. Foram observados resultados semelhantes quanto às variáveis: produtividade total e quantidade de frutos de tamanho: primeira, 1B e 1A em relação aos seis genótipos. Não houve diferença significativa a 5\%, pelo teste de Tukey, para: \% de frutos de coloração amarela, rosa e roxa; massa fresca de frutos amarelos e rosas; quantidade de frutos rosas e roxos, e também quantidade de frutos de tamanho $2 \mathrm{~A}$ e $3 \mathrm{~A}$ em relação a todos os genótipos avaliados. Os genótipos Rubi Gigante, Redondão, EC-3-0 e EC-L-7 podem ser recomendados para o cultivo no DF e região geoeconômica, se confirmarem a superioridade quando avaliados por um maior período e em diferentes condições ambientais.

Termos para indexação: melhoramento genético, Passiflora edulis,f. flavicarpa, características agronômicas.

\section{AGRONOMIC FEATURES OF SIX GENOTYPES OF PASSION FRUIT CULTIVATED IN DISTRITO FEDERAL, BRAZIL}

\begin{abstract}
This work had as objective to evaluate the agronomic features of six cultivated genotypes of passion fruit in the Distrito Federal. The experiment was developed in the experimental area of Fazenda Água Limpa (FAL) UnB, Brasilia-DF. The experimental design was randomized blocks with four useful repetitions, with six treatments, and seven plants per parcel. The six genotypes that had been evaluated were: Rubi Gigante, EC-3-0, EC-L-7, RC-3, Redondão and Gigante Amarelo. The analyzed parameters, in a period of six months ( 17 harvests) had been: total estimate productivity, quantity of fruits and coloration of the rind. The genotype Rubi Gigante had the biggest productivity $\left(16,69 \mathrm{t} / \mathrm{t} / \mathrm{ha}^{-1}\right)$, while the RC-3 the minor $\left(2,92 \mathrm{t} / \mathrm{t} / \mathrm{ha}^{-1}\right)$.It was observed similar results for the parameters: average productivity and quantity of fruit number of sizes: first, $1 \mathrm{~B}$ and $1 \mathrm{~A}$ in relation to all the evaluated genotypes. It did not have significant difference for the Tukey test at 5\% between the variables: \% of yellow, pink and purple fruits; fresh mass of yellow and pink fruits; amount of pink and purple fruits and also amount of size of the fruits $2 \mathrm{~A}$ and $3 \mathrm{~A}$ in relation to all the evaluated genotypes. The genotypes, Rubi Gigante, Redondão, EC-3-0 and EC-L-7 can be recommended for the culture in the DF and geo-economic region, if it was confirmed the superiority when evaluated by a bigger period and in different ambient conditions.
\end{abstract}

Index terms: genetic improvement, Passiflora eduli f. flavicarpa, agronomics features.

\footnotetext{
${ }^{1}$ (Trabalho 156-08). Recebido em: 13-06-2008. Aceito para publicação em: 30-04-2009.

${ }^{2}$ Eng. Agr. MSc., Bolsista CNPq, da Faculdade de Agronomia e Medicina Veterinária (FAV) da Universidade de Brasília (UnB).email: simonemiranda@unb.br,

${ }^{3}$ Eng. Agr. Dr., Prof. Associado I da FAV (UnB). Campus Universitário Darcy Ribeiro, Caixa Postal 04508, CEP. 70.910-970 BrasíliaDF. e-mail: peixoto@unb.br

${ }^{4}$ Eng. Agr. Dr.Pesquisador da Empresa Brasileira de Pesquisa Agropecuária e Professor Colaborador da Universidade de Brasília.C.P. 08223, CEP. 73310-970 Brasília -DF. e-mail: junqueir@embrapa.cpac.embrapa.br,

${ }^{5}$ Eng. Agr. MSc., Bolsista CNPq, da Faculdade de Agronomia e Medicina Veterinária (FAV) da Universidade de Brasília (UnB) email:cecelo19@yahoo.com
} 
A fruticultura brasileira é uma atividade agrícola de grande interesse para o País, devido a agregar características importantes e desejáveis, tais como a demanda de grande contingente de mão de obra no campo. Ao gerar empregos, esse ramo da agricultura assume importância para estudos que buscam alternativas viáveis para a mantença do homem no campo (MATTA, 2005).

$\mathrm{O}$ maracujazeiro-azedo tem-se destacado como cultura de acentuada expansão no Brasil, evidenciando a sua popularização no mercado interno nos diferentes segmentos de consumo, além de seu elevado potencial econômico para a região do Cerrado (ROSSI, 1998; FORTALEZA \& OLIVEIRA, 2002). Nosso País destaca-se como o maior produtor mundial de maracujá, seguido do Peru, Venezuela, África do Sul, Sri Lanka e Austrália. Apesar de ser o maior produtor de maracujá, a produtividade média brasileira de maracujá-azedo está em torno de $13 \mathrm{t} / \mathrm{ha}^{-1} / \mathrm{ano}^{-1}$, sendo considerada relativamente baixa, pois pesquisas já alcançaram $30-35 \mathrm{t} / \mathrm{ha}^{-1} / \mathrm{ano}^{-1}$ (DOS ANJOS et al., 2002). Junqueira et al. (1999) citam que um dos fatores responsáveis pela baixa produtividade da cultura do maracujazeiro é o cultivo de variedades inadequadas. A baixa produtividade é apontada por Ruggiero (2000) como um dos principais problemas da cultura, destacando a necessidade de pesquisas para melhor definir tecnologias de produção, capazes de proporcionar o aumento da produtividade, a possibilidade do aumento na sobrevida da cultura e a melhoria da qualidade dos frutos, que são essenciais para o sucesso do agricultor.

Dessa forma, a seleção de cultivares que apresentem boa produtividade e qualidade de seus frutos é de fundamental importância para o desenvolvimento da cultura no País. Seguindo este propósito, o presente trabalho objetivou avaliar o desempenho agronômico de seis genótipos de maracujazeiroazedo no Distrito Federal.

O experimento foi conduzido na Fazenda Água Limpa da Universidade de Brasília, cujo clima da região é do tipo $\mathrm{AW}$, caracterizado por chuvas concentradas no verão, de outubro a abril, e invernos secos, de maio a setembro. O delineamento foi o de blocos ao caso, com seis genótipos, sete plantas por parcela e quatro repetições. Foram testados os genótipos: EC-L-7 (Sul Brasil X Marília), EC-30 [(Marília X Rubi Gigante) X Marília], Gigante Amarelo (Redondão X MSC), RC-3 [Híbrido de seleção recorrente (Passiflora setacea X Marília)], Redondão (Seleção de material introduzido de Porto Rico em 1996) e Rubi Gigante (Roxo autraliano X Marília).

As mudas de maracujazeiro foram transplantadas para o campo, com cerca de 90 dias após a semeadura. O espaçamento utilizado foi de $2,75 \mathrm{~m}$ entre linhas e 2,5 m entre plantas, totalizando 1.454 plantas $\mathrm{ha}^{-1}$. A irrigação foi realizada por gotejamento, nos meses de setembro e outubro de 2004. O sistema de condução da lavoura foi por espaldeira vertical, com os mourões a uma distância de $5 \mathrm{~m}$ e 1 fio de arame liso a 1,8 $\mathrm{m}$ de altura em relação ao solo. As plantas foram conduzidas em haste única. Não foi realizada polinização artificial. As colheitas iniciais foram realizadas no período de 04 de dezembro de 2004 a 29 de junho de 2005 , num total de 17 colheitas. Os frutos colhidos foram pesados e classificados de acordo com o diâmetro equatorial do fruto em: Primeira $-\leq$ que $55 \mathrm{~mm} ; 1 \mathrm{~B}$ - entre 55 até $65 \mathrm{~mm} ; 1 \mathrm{~A}$ - entre 66 e $75 \mathrm{~mm} ; 2 \mathrm{~A}$ - entre 76 e $90 \mathrm{~mm}$ e $3 \mathrm{~A}$ - superior a $91 \mathrm{~mm}$, (Adaptação de OLIVEIRA, 2001 e RANGEL, 2002) e pela coloração da casca.

As variáveis analisadas foram: produtividade total estimada $\left(\mathrm{t} / \mathrm{ha}^{-1}\right)$ e quantidade total de frutos de tamanho primeira, $1 \mathrm{~A}, 1 \mathrm{~B}, 2 \mathrm{~A}$ e $3 \mathrm{~A}$; produtividade total estimada $\left(\mathrm{t} / \mathrm{ha}^{-1}\right)$, quantidade total , massa fresca $(\mathrm{g})$ e porcentagem de frutos amarelos, rosas e roxos.

Foram realizadas análises de variância (teste de F) para cada variável, bem como a comparação das médias por meio do teste de Tukey, a $5 \%$ de probabilidade de erro (GOMES, 1978), por meio do "software SANEST", de autoria de Zonta \& Machado (1995).

O genótipo EC-3-0 apresentou a maior produtividade total estimada de frutos $\left(30,92 \mathrm{t} / \mathrm{ha}^{-1} / \mathrm{ano}^{-1}\right)$ diferindo apenas do genótipo RC-3 (Tabela 1). Neste trabalho, o genótipo Redondão apresentou produtividade total de aproximadamente de 13,34 t/ $\mathrm{ha}^{-1} \mathrm{em} 17$ colheitas (seis meses), quantidade inferior à observada por Nascimento (2003) de 34,22 t/ ha ${ }^{-1} / \mathrm{ano}^{-1}$, após avaliar 61 colheitas, por Rangel (2002) de 29,06 t/ $\mathrm{ha}^{-1} / \mathrm{ano}^{-1}$ em 44 colheitas e por Oliveira (2001) de $17,00 \mathrm{t} /$ ha $^{-1}$ em 20 colheitas. Em relação à quantidade total de frutos produzidos, o genótipo EC-3-0 apresentou a maior quantidade, diferindo apenas do genótipo RC-3 (Tabela 2).

A maior produtividade média estimada de frutos classificados como primeira e apropriados para o mercado comercial para consumo in natura foi do genótipo Rubi Gigante de 7,36 t $/ \mathrm{ha}^{-1}$, diferenciandose significativamente apenas dos genótipos RC-3 e Redondão. Quanto aos frutos do tipo $1 \mathrm{~B}$, todos os genótipos avaliados, com exceção do genótipo RC3 , não apresentaram diferença significativa entre os mesmos. O genótipo EC-L-7 mostrou-se o mais produtivo para frutos $1 \mathrm{~A}$, quando comparado com 
os genótipos Gigante Amarelo e RC-3. O genótipo Redondão apresentou a maior produtividade total estimada de frutos $2 \mathrm{~A}$, não diferindo do genótipo EC3-0. O mesmo genótipo também foi o mais produtivo quanto aos frutos $3 \mathrm{~A}$, diferenciando-se do genótipo RE-0-3 (Tabela 1).

Entretanto, não foram verificadas diferenças significativas entre os genótipos avaliados quanto às variáveis: quantidade total de frutos dos tipos $2 \mathrm{~A} \mathrm{e}$ 3A (Tabela 2).

O genótipo EC-3-0 apresentou a maior produtividade total estimada de frutos amarelos, porém diferindo-se do genótipo RC-3. Quanto à produtividade total estimada de frutos rosa, o genótipo Rubi Gigante foi o que mais produziu frutos de coloração rosa, não se diferindo apenas do genótipo Gigante Amarelo, o mesmo também apresentou a maior produtividade total estimada de frutos roxos, diferenciou-se do genótipo Redondão.

Os genótipos EC-3-0, EC-L-7 e Redondão apresentaram as maiores quantidades totais de frutos amarelos, não diferindo significativamente entre si, com exceção do genótipo RC-3. Nas quantidades totais de frutos rosas e roxos, não ocorreram diferenças significativas entre os seis genótipos (Tabela 3).
Nas variáveis de massa fresca de frutos de coloração amarela e rosas, não foram observadas diferenças significativas entre os seis genótipos. Já a massa fresca dos frutos de coloração roxa, do genótipo Rubi Gigante, apresentou-se superior, diferenciando-se dos genótipos RE-0-3 e Redondão.

Não foram observadas diferenças significativas entre os genótipos quanto à porcentagem de frutos amarelos, rosas e roxos. Dos seis genótipos, todos apresentaram porcentagem superior a $71 \%$ de frutos de coloração amarela (Tabela 4). O genótipo Redondão apresentou a maior porcentagem de frutos amarelos, seguido do genótipo EC-3-0. Avaliando o mesmo genótipo, Oliveira (2001) observou valor superior $(98,08 \%)$ de frutos amarelos em 20 colheitas.

Para as condições e época de condução do experimento, concluiu-se que: os genótipos: Rubi Gigante, Redondão, EC-3-0 e EC-L-7 podem ser recomendados para o cultivo no Distrito Federal e região geoeconômica e que sejam conduzidos novos experimentos em diferentes locais desta região por um maior período de tempo para que seja estimado com maior precisão o potencial produtivo destes genótipos.

TABELA 1 - Produtividade total estimada de frutos (PT) em ( $\left.\mathrm{t} / \mathrm{ha}^{-1}\right)$, de tamanho primeira (FP), 1B (F1B), 1A(F1A), 2A (F2A) e 3A (F3A) de seis genótipos de maracujazeiro-azedo, durante seis meses de produção (17 colheitas), FAL-UnB, Brasília-DF, 2005.

\begin{tabular}{ccccccc}
\hline GENÓTIPO & PT $\left(\mathrm{t} / \mathrm{ha}^{-1}\right)$ & FP & F1B & F1A & F2A & F3A \\
\hline EC-3-0 & $15,46^{(1)} \mathrm{a}$ & $5,92 \mathrm{ab}$ & $5,54 \mathrm{a}$ & $3,35 \mathrm{ab}$ & $0,78 \mathrm{ab}$ & $0,01 \mathrm{ab}$ \\
EC-L-7 & $14,96 \mathrm{a}$ & $5,85 \mathrm{ab}$ & $5,36 \mathrm{a}$ & $3,86 \mathrm{a}$ & $0,51 \mathrm{~b}$ & $0,15 \mathrm{ab}$ \\
Gigante Amarelo & $10,92 \mathrm{a}$ & $6,39 \mathrm{ab}$ & $3,91 \mathrm{a}$ & $1,73 \mathrm{bc}$ & $0,35 \mathrm{~b}$ & $0,05 \mathrm{ab}$ \\
RC-3 & $2,92 \mathrm{~b}$ & $1,67 \mathrm{c}$ & $0,91 \mathrm{~b}$ & $0,34 \mathrm{c}$ & $0,13 \mathrm{~b}$ & $0,00 \mathrm{~b}$ \\
Redondão & $13,34 \mathrm{a}$ & $4,29 \mathrm{bc}$ & $4,39 \mathrm{a}$ & $3,26 \mathrm{ab}$ & $1,25 \mathrm{a}$ & $0,37 \mathrm{a}$ \\
Rubi Gigante & $16,69 \mathrm{a}$ & $7,36 \mathrm{a}$ & $5,82 \mathrm{a}$ & $3,17 \mathrm{ab}$ & $0,41 \mathrm{~b}$ & $0,01 \mathrm{ab}$ \\
\hline CV $(\%)$ & 20.753 & 23,502 & 23,645 & 27,996 & 24,546 & $0,771^{(2)}$ \\
\hline
\end{tabular}

("Para cada variável, médias seguidas pela mesma letra, na coluna, não diferem entre si, pelo teste de Tukey, ao nível de $5 \%$ de significância. ${ }^{(2)}$ Variável transformada para raiz $(\mathrm{x}+10)$.

TABELA 2 - Quantidade total de frutos (QFT), do tipo primeira (QFP), 1B (QF1B), 1A(QF1A), 2A (QF2A), 3A (QF3A) de seis genótipos de maracujazeiro-azedo, durante seis meses de produção (17 colheitas), FAL-UnB, Brasília-DF, 2005.

\begin{tabular}{ccccccc}
\hline GENÓTIPOS & QFT & QFP & QF1B & QF1A & QF2A & QF3A \\
\hline EC-3-0 & $217.98 \mathrm{a}$ & $74.25^{(1)} \mathrm{ab}$ & $38.98 \mathrm{a}$ & $24.53 \mathrm{ab}$ & $3.86 \mathrm{a}$ & $1.22 \mathrm{a}$ \\
EC-L-7 & $158.99 \mathrm{ab}$ & $76.35 \mathrm{ab}$ & $45.43 \mathrm{a}$ & $33.69 \mathrm{a}$ & $3.35 \mathrm{a}$ & $1.99 \mathrm{a}$ \\
Gigante Amarelo & $133.01 \mathrm{ab}$ & $82.34 \mathrm{ab}$ & $31.81 \mathrm{a}$ & $16.34 \mathrm{bc}$ & $2.40 \mathrm{a}$ & $1.73 \mathrm{a}$ \\
RC-3 & $48.01 \mathrm{~b}$ & $16.98 \mathrm{c}$ & $7.47 \mathrm{~b}$ & $10.07 \mathrm{c}$ & $2.24 \mathrm{a}$ & $1.99 \mathrm{a}$ \\
Redondão & $141.45 \mathrm{ab}$ & $53.37 \mathrm{bc}$ & $38.37 \mathrm{a}$ & $27.91 \mathrm{ab}$ & $6.95 \mathrm{a}$ & $2.74 \mathrm{a}$ \\
Rubi Gigante & $164.13 \mathrm{ab}$ & $91.28 \mathrm{a}$ & $44.99 \mathrm{a}$ & $26.19 \mathrm{ab}$ & $2.83 \mathrm{a}$ & $0.74 \mathrm{a}$ \\
\hline CV $(\%)$ & $19.903^{(2)}$ & 24.066 & 22.301 & 24.295 & 31.707 & $3.938^{(2)}$
\end{tabular}

(1)Para cada variável, médias seguidas pela mesma letra, na coluna, não diferem entre si, pelo teste de Tukey, ao nível de $5 \%$ de significância. ${ }^{(2)}$ Variável transformada para raiz $(\mathrm{x}+10)$. 
TABELA 3 - Quantidade total de frutos de coloração amarela (QFAM), rosa (QFRS) e roxa (QFRX) e massa fresca (g) de frutos de coloração amarela (MFAM), rosa (MFRS) e roxa (MFRX) de seis genótipos de maracujazeiro-azedo, durante seis meses de produção (17 colheitas), FALUnB, Brasília-DF, 2005.

\begin{tabular}{ccccccc}
\hline GENÓTIPOS & QFAM & QFRS & QFRX & MFAM & MFRS & MFRX \\
\hline EC-3-0 & $200.25^{(1)} \mathrm{a}$ & $6.408 \mathrm{a}$ & $10.25 \mathrm{a}$ & $206,59 \mathrm{a}$ & $76,97 \mathrm{a}$ & $113,37 \mathrm{ab}$ \\
EC-L-7 & $142.13 \mathrm{a}$ & $6.89 \mathrm{a}$ & $8.12 \mathrm{a}$ & $210,59 \mathrm{a}$ & $94,28 \mathrm{a}$ & $98,54 \mathrm{ab}$ \\
Gigante Amarelo & $114.38 \mathrm{ab}$ & $11.08 \mathrm{a}$ & $6.67 \mathrm{a}$ & $189,12 \mathrm{a}$ & $120,14 \mathrm{a}$ & $64,55 \mathrm{ab}$ \\
RC-3 & $30.29 \mathrm{~b}$ & $8.78 \mathrm{a}$ & $7.169 \mathrm{a}$ & $147,77 \mathrm{a}$ & $48,18 \mathrm{a}$ & $28,50 \mathrm{~b}$ \\
Redondão & $138.60 \mathrm{a}$ & $3.35 \mathrm{a}$ & $0.939 \mathrm{a}$ & $197,04 \mathrm{a}$ & $66,89 \mathrm{a}$ & $21,69 \mathrm{~b}$ \\
Rubi Gigante & $114.91 \mathrm{ab}$ & $29.19 \mathrm{a}$ & $17.088 \mathrm{a}$ & $196,54 \mathrm{a}$ & $169,16 \mathrm{a}$ & $181,58 \mathrm{a}$ \\
\hline CV $(\%)$ & $20.280^{(2)}$ & $30.912^{(2)}$ & $22.399^{(2)}$ & 16.445 & $34.603^{(2)}$ & $30.431^{(2)}$
\end{tabular}

(1) Para cada variável, médias seguidas pela mesma letra, na coluna, não diferem entre si, pelo teste de Tukey, ao nível de $5 \%$ de significância. ${ }^{(2)}$ Variável transformada para raiz $(\mathrm{x}+10)$.

TABELA 4 - Porcentagem de frutos de coloração amarela (\%FAM), rosa (\%FRS) e roxa (\%FRX) e produtividade total estimada de frutos de coloração amarela (PAM) em $\left(\mathrm{t} / \mathrm{ha}^{-1}\right)$, rosa (PRS) e roxa (PRX) de seis genótipos de maracujazeiro-azedo, durante seis meses de produção (17 colheitas), FAL-UnB, Brasília-DF, 2005.

\begin{tabular}{ccccccc}
\hline GENÓTIPOS & \%FAM & \%FRS & \%FRX & PAM & PRS & PRX \\
\hline EC-3-0 & $91,08^{(1)} \mathrm{a}$ & $4,17 \mathrm{a}$ & $4,40 \mathrm{a}$ & $13,32 \mathrm{a}$ & $0,75 \mathrm{~b}$ & $1,46 \mathrm{ab}$ \\
EC-L-7 & $89,31 \mathrm{a}$ & $4,65 \mathrm{a}$ & $5,27 \mathrm{a}$ & $14,08 \mathrm{a}$ & $0,82 \mathrm{~b}$ & $0,85 \mathrm{ab}$ \\
Gigante Amarelo & $86,31 \mathrm{a}$ & $8,15 \mathrm{a}$ & $5,03 \mathrm{a}$ & $10,53 \mathrm{a}$ & $1,23 \mathrm{ab}$ & $0,66 \mathrm{ab}$ \\
RC-3 & $77,47 \mathrm{a}$ & $9,63 \mathrm{a}$ & $8,52 \mathrm{a}$ & $2,40 \mathrm{~b}$ & $0,18 \mathrm{~b}$ & $0,38 \mathrm{ab}$ \\
Redondão & $96,66 \mathrm{a}$ & $2,55 \mathrm{a}$ & $0,74 \mathrm{a}$ & $13,08 \mathrm{a}$ & $0,38 \mathrm{~b}$ & $0,10 \mathrm{~b}$ \\
Rubi Gigante & $71,16 \mathrm{a}$ & $17,19 \mathrm{a}$ & $10,31 \mathrm{a}$ & $10,98 \mathrm{a}$ & $3,59 \mathrm{a}$ & $2,08 \mathrm{a}$ \\
\hline CV (\%) & 20.095 & $26.927^{(2)}$ & $18.314^{(2)}$ & 18.710 & $5.148^{(2)}$ & $3.504^{(2)}$ \\
\hline
\end{tabular}

${ }^{(1)}$ Para cada variável, médias seguidas pela mesma letra, na coluna, não diferem entre si, pelo teste de Tukey, ao nível de $5 \%$ de significância. ${ }^{(2)}$ Variável transformada para raiz $(x+10)$.

\section{REFERÊNCIAS}

DOS ANJOS, J.R.N.; JUNQUEIRA, N.T.V.; CHARCHAR, M.J.A. Levantamento do Passion fruit Woodiness Vírus em maracujazeiro-azedo no Cerrado do Brasil central. In: CONGRESSO DE FRUTICUlTURA, 17., 2002. Belém. Anais... Belém: SBF, 2002. p. 520.

FORTALEZA, J.M.; OLIVEIRA, A.T. Características físico-químicas do maracujazeiro-azedo, cultivado sob três níveis de adubação potássica no Distrito Federal. In: CONGRESSO BRASILEIRO DE FRUTICULTURA, 17., 2002. Belém. Anais... Belém: SBF, 2002. p. 650.

GOMES, F.P. Curso de estatística experimental. 8.ed. São Paulo: Nobel, 1978. 430p.
JUNQUEIRA, N. T. V.; ICUMA, I. M.; VERAS, M. C. M.; OLIVEIRA, M. A. S.; DOS ANJOS, J. R. N. Cultura do maracujazeiro. In: SILVA, J.M. de M. (Ed.). Incentivo a fruticultura no Distrito Federal: manual de fruticultura. Brasília: COOLABORA, 1999. p. $42-52$.

MATTA, F.P. Mapeamento de QRL para Xanthomonas axonopodis pv. passiflorae em maracujáazedo (Passiflora edulis Sims f. flavicarpa Deg.). 2005. 230 f. Tese (Doutorado) - Escola Superior de Agricultura Luis de Queiroz, Universidade de São Paulo, Piracicaba, 2005.

NASCIMENTO, A.C. Produtividade, incidência e severidade de doenças em nove genótipos de maracujazeiro-amarelo sob três níveis de adubação potássica no Distrito Federal. 2003. 133 f. Dissertação (Mestrado em Ciências Agrárias) - Faculdade de Agronomia e Medicina Veterinária, Universidade de Brasília, Brasília, 2003. 
OLIVEIRA, A.T.de. Produtividade e avaliação da incidência e severidade de doenças em frutos de nove genótipos de maracujazeiro-azedo cultivados sob influência de adubação potássica no Distrito Federal. 2001. 83 f. Dissertação (Mestrado em Ciências Agrárias) - Faculdade de Agronomia e Medicina Veterinária, Universidade de Brasília, Brasília, 2001.

RANGEL, L.E.D. Desempenho agronômico de nove genótipos de maracujazeiro-amarelo cultivados sob três diferentes níveis de adubação potássica no Distrito Federal. 2002. 46 f. Dissertação (Mestrado em Ciências Agrárias) - Faculdade de Agronomia e Medicina Veterinária, Universidade de Brasília, Brasília, 2002.
ROSSI, A.D. Comercialização do maracujá. In: SIMPÓSIO BRASILEIRO SOBRE A CULTURA DO MARACUJAZEIRO, 5., 1998, Jaboticabal. Anais.... Jaboticabal: Funep, 1998. p. 279-287.

RUGGIERO, C. Situação da cultura do maracujazeiro no Brasil. Informe Agropecuário, Belo Horizonte, v.21, n. 206, p 5-9, 2000.

ZONTA, E. P.; MACHADO, A. A. Sistema de análises estatísticas (SANEST) para microcomputadores. In: SIMPÓSIO DE ESTATÍSTICA APLICADA À EXPERIMENTAÇÃO, 1995, Piracicaba. Anais... Campinas: Fundação Cargill, 1995. p.17-18. 